\title{
ENHANCED OPTICAL COOLING OF ION BEAMS FOR LHC*
}

\author{
E.G.Bessonov\#, M.V.Gorbunkov, Lebedev Phys. Inst. RAS, Moscow, \\ Russia, A.A.Mikhailichenko, Cornell University, Ithaca, NY, U.S.A.
}

(Dated: February 9, 2020)

\begin{abstract}
The possibility of the enhanced optical cooling (EOC) of Lead ions in LHC is investigated. Nonexponential feature of cooling and requirements to the ring lattice, optical and laser systems are discussed. Comparison with optical stochastic cooling (OSC) is represented.

PACS numbers: 29.20.Dh, 07.85.Fv, 29.27.Eg
\end{abstract}

\section{INTRODUCTION}

In original OSC with usage of quadrupole wiggler as a pickup [1], particles with small betatron amplitude do not make an input into signal generation (radiation), so they are not heating the beam. In contrast, in EOC method such selective action achieved by usage of movable screens. These screens located on image plane of optical system having radiating beam as source. Motion realized with the help of fast electro-optical elements driven by external voltage. As a result of this selection the ions with extreme deviations of dynamic variables keep the neighboring ions undisturbed in the first approximation. By this way the number of the particles in the bandwidth, which defines the damping time can be reduced drastically. Some detailed schemes of EOC were suggested in [2]-[4]. Below we consider EOC of fully stripped Lead ions in LHC as example.

\section{THE SCHEME OF COOLING}

The EOC method uses a pickup undulator and one or more kicker undulators installed in different straight sections of a storage ring. The distance determined by a betatron phase advance $(2 p-1) \pi$ between the pickup and the first kicker undulator and $2 p^{\prime} \pi$ between each of the following kicker undulators; where $p, p^{\prime}=1,2,3 \ldots$ Undulator Radiation Wavelets (URW), emitted by ions in the pickup undulator, transferred by optical system to the movable screen located on the image plane. Here the undesirable part of radiation, corresponding to small betatron amplitudes, is cut. Residual fraction or URW amplified in optical amplifier and pass together with the ions through the followed kicker undulators.

\section{THE RATE OF COOLING}

The change of the square of the amplitude of betatron oscillations of an ion, caused by sudden energy change $\delta E$

\footnotetext{
*Supported by RFBR under grant No 05-02-17162 and by NSF.

\# bessonov@x4u.lebedev.ru
}

in a kicker undulator is determined in smooth approximation by

$$
\delta A_{x}^{2}=-2 x_{\beta, k} \delta x_{\eta}+\left(\delta x_{\eta}\right)^{2}
$$

where $x_{\beta, k}$ is the ion deviation from it's closed orbit in the kicker undulator; $\delta x_{\eta}=\eta_{x} \beta^{-2}(\delta E / E)$ is the change of it's closed orbit position; $\eta_{x}$ is the dispersion function in the storage ring; $\beta$ is the normalized velocity. In the approximation $\left|\delta x_{\eta}\right|<2\left|x_{\beta, k}\right|<2 A_{x}$ both the betatron amplitude and the position of the closed orbit will be decreased, if the values $x_{\beta, k}<0, \delta x_{\eta}<0$. It follows that to cool the ion beam the screen in the optical system must open the pass for URWs emitted by extreme ions entering the pickup undulator with higher energy and betatron deviations $x_{\beta, p}>0$ from theirs closed orbits. After that the screen will open images of ions with lower and lower energies until the optical system must be switched off. Then the cooling process can be repeated. So the EOC is going simultaneously both in the longitudinal and transverse degrees of freedom.

Optical lengths between pickup and kicker undulators should be picked up so that to inject ions in the kicker undulators at decelerating phases of their own URWs.

The total energy of the undulator radiation (UR) emitted by a relativistic ion traversing an undulator with magnetic field $B$ is given by

$$
E_{t o t}=\frac{2}{3} r_{i}^{2} \overline{B^{2}} \gamma^{2} L_{u}
$$

where $\overline{B^{2}}$ is an average square of magnetic field along the undulator period $\lambda_{u} ; r_{i}=Z^{2} e^{2} / M_{i} c^{2}$ is the classical radius of the ion; $e, M_{i}$ are the electron charge and ion mass respectively; $Z$ is the atomic number, $L_{u}=M \lambda_{u} ; M$ is the number of undulator periods; $\gamma$ is the relativistic factor. For a plane harmonic undulator $\overline{B^{2}}=B_{0}^{2} / 2$, where $B_{0}$ is the peak of the undulator field. For helical undulator $\overline{B^{2}}=B_{0}^{2}$. The spectrum of the first harmonic of the UR is $d E_{1} / d \xi=E_{1} f(\xi)$, where $E_{1}=E_{\text {tot }} /\left(1+K^{2}\right)$, $K=Z e \sqrt{\overline{B^{2}}} \lambda_{u} / 2 \pi M_{i} c^{2}, f(\xi)=3 \xi\left(1-2 \xi+2 \xi^{2}\right), \xi=$ $\lambda_{1, \min } / \lambda_{1}, \lambda_{1 \text { min }}=\left.\lambda_{1}\right|_{\theta=0},(0 \leq \xi \leq 1), \int f(\xi) d \xi=1$, $M>>1, \lambda_{1}=\lambda_{u}\left(1+K^{2}+\bar{\vartheta}^{2}\right) / 2 \gamma^{2}$ is the wavelength of the first harmonic of the UR, $\vartheta=\gamma \theta ; \theta$, the azimuth angle. 
The number of the equivalent photons in the URW in the suitable for cooling frequency range $(\Delta \omega / \omega)_{c}=$ $1 / 2 M$ and angular range $\Delta \vartheta=\sqrt{\left(1+K^{2}\right) / 2 M}$

$$
N_{p h}=\Delta E_{1} / \hbar \omega_{1 \max }=\pi \alpha Z^{2} K^{2},
$$

where $\Delta E_{1}=\left(d E_{1} / d \omega\right) \Delta \omega=3 E_{\text {tot }} / 2 M\left(1+K^{2}\right)$, $\omega_{1 \max }=2 \pi c / \lambda_{1 \min }, M=L_{u}\left(1+K^{2}\right) / 2 \gamma^{2} \lambda_{1 \min }$. An aperture or filters must be used in the optical system to select a portion of URW in this frequency range for resonance interaction of ions with their URWs in kicker undulators.

Below we accept a Gaussian distribution for the URW, its Rayleigh length $Z_{R}=4 \pi \sigma_{w}^{2} / \lambda_{1 \text { min }}=L_{u} / 2$, the rms waist size $\sigma_{w}^{=} \sqrt{L_{u} \lambda_{1 \min } / 8 \pi}$. In this case the rms electric field strength $E_{w}$ of the wavelet in the kicker undulator

$$
E_{w}=\sqrt{2 \Delta E_{1} / \sigma_{w}^{2} \lambda_{1 \min }}=8 \sqrt{\pi} r_{i} \gamma^{3} \sqrt{\overline{B^{2}}} / L_{u}\left(1+K^{2}\right)^{3 / 2} .
$$

The rate of the energy loss for ions in the amplified URW is

$$
\begin{gathered}
P_{\text {loss }}=e Z E_{w} L_{u} \beta_{\perp m} f N_{k i c k} \sqrt{\alpha_{a m p l}}= \\
8 \sqrt{\pi} e Z r_{i} f \gamma^{2} N_{k i c k} \sqrt{\alpha_{a m p l} \cdot \overline{B^{2}}} K /\left(1+K^{2}\right)^{3 / 2}
\end{gathered}
$$

where $\beta_{\perp}=K / \gamma ; f$ is the revolution frequency; $N_{k i c k}$ is the number of kicker undulators; $\alpha_{a m p l}$ is the gain in optical amplifier.

The damping time for the ion beam in the longitudinal degree of freedom is

$$
\tau=\sigma_{E} / P_{l o s s},
$$

where $\sigma_{E}$ is the energy spread of the ion beam.

According to (6), the damping time for EOC is proportional to the energy spread of the beam which is much less then the energy of ions included in similar expression for damping time controlled by Robinson's damping criterion. Moreover, because of the non-exponential decay of both energy and angular spreads of the beam the degree of cooling of ion beams for EOC is much higher than 1 /e reduction of these parameters.

Note that the higher the dispersion function and the less the beta function at the location of the kicker undulator the higher the rate of damping of betatron oscillations. In this case low energy jumps of ions lead to large jumps of closed orbits and near the same large jumps of betatron oscillation amplitudes.

\section{STOCHASTIC PROCESSES IN THE EOC}

URW of one ion does not disturb trajectories of other ions if an average distance between ions in the longitudinal direction is more, than the URW's length, $M \lambda_{U R, 1}$, and the transverse dimensions of the URW's in kicker undulators are overlapped and higher then the transverse total (dispersion + betatron) dimensions of the being cooled ion beam. This case is named "single ion in the sample". It corresponds to the beam current

$$
i<i_{c}=\frac{Z e c}{M \lambda_{1 \min }}=\frac{4.8 \cdot 10^{-9} Z}{M \lambda_{1 \min }[\mathrm{cm}]}[A] .
$$

If $i>i_{c}$ amplified URWs do not disturb the energy spread and amplitudes of betatron oscillations of other's ions of the beam in the first approximation and change them in the second one because of a stochasticity of the initial phases of ions in other's URWs. Stochasticity limits the degree of cooling.

Open ions of the beam loose their energies up to the moment when they will be displayed inward to the distances corresponding to overlapping their URWs by the stopped screen. After this time all ions will stay at the threshold energy with the equilibrium energy spread and the spread of amplitudes of betatron oscillations [3]

$$
\left(\sqrt{\overline{A_{x}^{2}}}\right)_{e q}=\left(\sqrt{\overline{x_{\eta}^{2}}}\right)_{e q}=\frac{1}{2}\left|\delta x_{\eta}\right|\left(n_{c}+1+n_{n}\right)
$$

determined by the average jump of the ion energy $\Delta E=$ $P_{\text {loss }} \sqrt{n_{c}+1+n_{n}} / f$, where $\delta x_{\eta}=\eta_{x} \beta^{-2}\left(P_{\text {loss }} / E f\right)$, $n_{c}=i / i_{c}$ is the number of ions in a sample; $n_{n}=$ $N_{n} / N_{p h} ; N_{n}$, the number of noise photons in the URW sample at the amplifier front end.

\section{STORAGE RING LATTICE REQUIREMENTS}

The relative phase shifts of ions in their URWs radiated in the pickup undulator and displaced to the entrance of kick undulators depend on theirs energy and amplitude of betatron oscillations. If we assume that the longitudinal shifts of URWs $\Delta l<\lambda_{U R} / 2$, then the amplitudes of betatron oscillations, transverse horizontal emittance of the beam, in the smooth approximation, and the energy spread of the beam must not exceed the values

$$
\begin{gathered}
A_{x}<<A_{x, \lim }=\frac{\lambda_{1 \mathrm{~min}} \sqrt{\lambda_{\text {bet }}}}{\pi}, \quad \varepsilon_{x}<2 \lambda_{U R 1 \mathrm{~min}}, \\
\frac{\Delta \gamma}{\gamma}<\left(\frac{\Delta \gamma}{\gamma}\right)_{\lim }=\frac{\beta^{2}}{\eta_{c}} \frac{\lambda_{1 \mathrm{~min}}}{\lambda_{\text {bet }}},
\end{gathered}
$$

where $\lambda_{x, b e t}=C / v_{x} ; \mathrm{C}$ is the circumference of the ring, $v_{x}$, the tune; $\eta_{c}=\alpha_{c}-\gamma^{-2}$ and $\alpha_{c}$ are local slip and momentum compaction factors between undulators.

Strong limitations (9) to the energy spread can be overcame if, according to the decrease of the high energy edge of the being cooled beam, a change in time of optical paths of URWs is produced. Special elements in storage 
ring lattices (short inverted dipoles, quadrupole lenses et al.) to decrease the slip [5-8] can be used as well. With cooling of fraction of the beam at a time only, the lengthening problem diminishes also as the $\Delta E / E$ now stands for the energy spread in the part of the beam which is under cooling at the moment.

\section{OPTICAL SYSTEM FOR EOC}

The power of the optical amplifier is equal to the power of the amplified URWs plus the noise power

$$
P_{\text {ampl }}=\varepsilon_{\text {sample }} \cdot f \cdot N_{i}+P_{n},
$$

where $\varepsilon_{\text {sample }}=\hbar \omega_{1, \max } N_{p h} \alpha_{a m p l}$ is the energy in a sample; $N_{i}$, the number of ions in the ring. The bunch spacing in LHC $(45 \mathrm{~m})$ is much bigger than the bunch length $(\sim 5-10 \mathrm{~cm})$. The same time structure of the OPA must be used. The energy of OPA emitted for the damping time is proportional to the initial energy spread of the ion beam. It can be decreased by decreasing peak RF voltage or by increasing the number of bunches.

The space resolution of the ion beam is [3]

$$
\delta x_{\text {res }} \simeq 1.22 \lambda_{1 \mathrm{~min}} / \Delta \theta=1.22 \sqrt{\lambda_{1 \min } L_{u}} .
$$

The transverse selectivity of radiation (movable screen) can be arranged with help of electro-optical elements. These elements contain crystals, which change their refraction index while external voltage applied. This technique is well known in optics [9]. In simplest case the sequence of electro-optical deflector and a diaphragm followed by optical lenses, allow controllable selection of radiation generated by different parts of the beam.

Example 1. EOC of fully stripped ${ }_{207}^{82} \mathrm{~Pb}$ ion beam in the CERN LHC at the injection energy $M_{i} c^{2} \gamma=$ $=36.9 \mathrm{TeV}$. The parameters of the LHC: circumference $\mathrm{C}=27 \mathrm{~km}, f=1.1 \cdot 10^{4}, v_{x}=64.28, \alpha_{c}=3.23 \cdot 10^{-4}$, $i=6.28 \mathrm{~mA}, \eta_{c}=3.18 \cdot 10^{-4}, N_{i}=4.1 \cdot 10^{10}, \gamma=190.5$, $\Delta \gamma / \gamma=3.9 \cdot 10^{-4}$, normalized emittance $\epsilon_{x, n}=1.4_{\mu} m$, beta and dispersion functions at the kicker undulator $\beta_{x}=25.0 \mathrm{~m}, \eta_{x}=2.0 \mathrm{~m}$, betatron beam size at pickup undulator $\sigma_{x, 0}=0.43 \mathrm{~mm}$, dispersion beam size $\sigma_{\eta, 0}=0.95 \mathrm{~mm}$, total beam size $\sigma_{b, 0}=1.1 \mathrm{~mm}$.

One pickup one kick helical undulator with parameters $\sqrt{\overline{B^{2}}}=10^{5} \mathrm{Gs}, \lambda_{u}=4 \mathrm{~cm}, M=300$ and two optical parametric amplifiers (OPA) with gains $\sim 10^{4}$ are used. The total gain goes to be $\alpha_{a m p l}=10^{8}$.

In this case: $M_{i} c^{2}=1.94 \cdot 10^{11} \mathrm{eV}, N_{p h}=1.01 \cdot 10^{-2}$, $r_{i}=4.96 \cdot 10^{-15} \mathrm{~cm}, i_{c}=0.024 \mathrm{~mA}, N_{c}=i_{c} /$ ef $=1.7 \cdot 10^{8}$, $\lambda_{1 \min }=5.5 \cdot 10^{-5} \mathrm{~cm}, \sigma_{w}=0.51 \mathrm{~mm}, K=0.0081, E_{w} \cong$ $1.22 \cdot 10^{-2} \mathrm{~V} / \mathrm{cm}, P_{\text {loss }}=5.61 \cdot 10^{6} \mathrm{eV} / \mathrm{sec}, \tau=42.8 \mathrm{~min}$, $P_{a m p l}=164 \mathrm{~W}, \delta x_{\eta}=2.76 \cdot 10^{-9} \mathrm{~cm}, \lambda_{x, \text { bet }}=414.7$ $\mathrm{m}, A_{x, \lim }=5 \mathrm{~mm},(\Delta \gamma / \gamma)_{\lim }=4.17 \cdot 10^{-6}, \delta x_{\text {res }}=$ $3.14 \mathrm{~mm}, n_{c}=241, n_{n}=99$, equilibrium beam dimensions $\left(\overline{A_{x}^{2}}\right)_{e q}^{1 / 2}=\left(\overline{x_{\eta}^{2}}\right)_{e q}^{1 / 2}=3.45 \cdot 10^{-7} \mathrm{~cm}$,
Damping time of an ion beam, according to (5), (6), is $\tau=\sigma_{E} / P_{\text {loss }} \mid \tilde{K}_{<0.5} \sigma_{E} / N_{k i c k} K \gamma^{2}$. If $\lambda_{1 \mathrm{~min}}, \sigma_{E}$ and $\overline{B^{2}}$ are constants, then $\tau \sim 1 / \gamma^{4}$ and the power of the optical amplifier $P_{a m p l} \sim N_{p h} N_{i} \sim N_{i} \gamma^{4}$ Damping time can be decreased by using beams with smaller initial energy spread $\sigma_{E}$, many kicker undulators $N_{k i c k}>1$ and higher gain of optical amplifiers.

Example 2. EOC of fully stripped ${ }_{207}^{82} P b$ ion beam in the CERN LHC at the energy $\gamma=953, \Delta \gamma / \gamma=1.3$. $10^{-4}, N_{i}=10^{8}$, betatron beam size $\sigma_{x, 0}=4.3 \mathrm{~mm}$, dispersion beam size $\sigma_{\eta, 0}=9.5 \mathrm{~mm}$, total beam size $\sigma_{b, 0}=11 \mathrm{~mm}$ at pickup undulator. One pickup and one kick undulator with parameters $\sqrt{\overline{B^{2}}}=10^{5} \mathrm{Gs}, \lambda_{u}=1$ $\mathrm{m}, K=0.202, M=12$ and OPAs identical to ones of the example 1 are used.

In this case: $N_{p h}=6.29, \lambda_{1 \min }=5.5 \cdot 10^{-5} \mathrm{~cm}, \sigma_{w}=$ $0.51 \mathrm{~mm}, \delta x_{\text {res }}=3.14 \mathrm{~mm}, E_{w} \cong 1.44 \mathrm{~V} / \mathrm{cm}, P_{\text {loss }}=$ $3.3 \cdot 10^{9} \mathrm{eV} / \mathrm{sec}, P_{a m p l}=249 \mathrm{~W}, \delta x_{\eta}=3.25 \cdot 10^{-6} \mathrm{~cm}, \tau=$ $4.36 \mathrm{sec}$, the equilibrium beam dimensions $\left(\overline{A_{x}^{2}}\right)_{e q}^{1 / 2}=$ $\left(\overline{x_{\eta}^{2}}\right)_{e q}^{1 / 2}=1.22 \cdot 10^{-6} \mathrm{~cm}$.

In these examples initial transverse beam dimensions $\sigma_{x, 0}<A_{x, \lim }$. It means that there is no problem with the dependence of phase shifts of ions on their amplitudes. At the same time there is a necessity in special elements in storage ring lattices to decrease the slip.

We were forced to decrease the number of ions in the second example to reach acceptable power of OPA. It can be increased if the gain of OPA will be decreased. At that damping time will be increased.

The space resolution of the ion beam and that is why its final dimensions are limited by a value $\delta x_{r e s}=3.14$ $\mathrm{mm}$, which is larger then the initial beam dimension $\sigma_{b, 0}=1.1 \mathrm{~mm}$ in the example 1 . It does not permit to reach small equilibrium beam dimensions and means that we must increase the initial beam dimensions by increasing the dispersion and beta functions in the location of the pickup undulator or, according to (11), decrease the length of the undulator and using more short $\lambda_{1 \mathrm{~min}}$ to increase the resolution. High beam size in the pickup undulator can be arranged by appropriate betatron function at this place.

Cooling of ion beams is produced in the RF bucket. In this case the screen must be moved to the position of the image of the equilibrium orbit and stopped at this position. Cooling cycles must be repeated periodically. The length of the URW bunch can be less then ion one. In this case only overlapped parts of ion and URW beams will interact. Despite of this all ion beam will be cooled as ions take part in phase oscillations.

\section{CONCLUSION}

We considered EOC of ion beams in LHC storage ring. Details of EOC for bunched beam will be presented in 
a separate paper. The gain of the OPAs can be higher then considered in the paper. That is why the damping time is limited by the maximum achievable power of the OPA and number of kicker undulators.
[1] A.Mikhailichenko, M.Zolotorev, Phys.Rev.Lett.71: 4146$4149,1993$.

[2] E.G.Bessonov, physics/0404142

[3] E.G.Bessonov, A.A.Mikhailichenko, Proc. PAC05, May 16-20, 2005, Knoxville, Tennessee, USA. http://accelconf.web.cern.ch/accelconf/p05/PAPERS/ TPAT086.PDF.

[4] E.G.Bessonov, A.A.Mikhailichenko, A.V.Poseryaev, Physics of the Enhanced optical cooling of particle beams in storage rings, http://arxiv.org/abs/physics/0509196
[5] C.Pellegrini and D.Robin, Nucl. Instr. Meth. A301, 27 (1991); Proc. IEEE Part. Accel. Conf., San Francisco, p. 398 (1991).

[6] M.Berz, Part. Acc. V.24, p.109, 1989.

[7] E.Forest, M.Berz, J.Irwin, Part. Acc. V.24, p.91 (1989).

[8] Klaus G.Steffen, High Energy Beam Optics, Interscience publishers, NY-London-Sydney, 1965.

[9] V.J.Fowler, J.Schlafer, Applied Optics Vol.5, N10, 1657 (1966). 